\title{
An Extraordinary Case of Idiopathic Chylothorax in a Rural Setting
}

\author{
Kevin Chow Kuan Yee1, Ong Tze Hern'1, Ng Zxai Ching1, Naganathan Pillai', Adelyn Henry², \\ Lee Tiong Chan ${ }^{2}$
}

\author{
${ }^{1}$ Monash University Malaysia, Bandar Sunway, Malaysia \\ ${ }^{2}$ Department of Internal Medicine, Hospital Segamat, Segamat, Malaysia \\ Email: pillainag@gmail.com
}

How to cite this paper: Yee, K.C.K., Hern, O.T., Ching, N.Z., Pillai, N., Henry, A. and Chan, L.T. (2018) An Extraordinary Case of Idiopathic Chylothorax in a Rural Setting. Open Access Library Journal, 5: e4924.

https://doi.org/10.4236/oalib.1104924

Received: September 18, 2018

Accepted: October 27, 2018

Published: October 30, 2018

Copyright ( 2018 by authors and Open Access Library Inc.

This work is licensed under the Creative Commons Attribution International License (CC BY 4.0).

http://creativecommons.org/licenses/by/4.0/

\section{Open Access}

\begin{abstract}
Chylothorax is a rare cause of pleural effusion, which can be divided into traumatic and non-traumatic causes. Often, the cause of a non-traumatic chylothorax is challenging. We report a young gentleman with idiopathic chylothorax who was diagnosed in a rural hospital with limited resources. This was confirmed on clinical presentation, pleural fluid analysis, CECT imaging and a diagnostic lymphangiogram. Contrast enhanced imaging did not reveal any secondary pathology. The chylothorax was subsequently drained and he was referred to a tertiary centre for definitive treatment. Managing chylothorax can be challenging in a rural setting due to the lack of relevant expertise and facilities. A timely diagnosis and treatment of chylothorax would require appropriate investigations to elucidate any underlying pathology and the lymphangiogram as a useful tool in diagnosis.
\end{abstract}

\section{Subject Areas \\ Internal Medicine}

\section{Keywords}

Chylothorax, Pleural Effusion, Lymphangiogram, Rural Setting

\section{Introduction}

Chylothorax is an uncommon cause of pleural effusion characterised by the leakage of chyle, which is a complex fluid originated from the digestive system consisting of lipid, protein, electrolytes and lymphocytes, into the pleural space. While the epidemiology of chylothorax remains elusive, its prevalence attributed directly to cardiothoracic surgeries is $0.2 \%-1 \%$, with an estimated $10 \%$ mortal- 
ity and morbidity rates [1]. The aetiology of this condition can be broadly classified into two major groups: traumatic and non-traumatic chylothoraces. The traumatic causes, which encompass thoracic surgical procedures, for instance lobectomy and invasive procedures or other forms of traumatic injuries, seemed to be as common as the non-traumatic ones. In contrast, the non-traumatic causes include neoplasm, infection, autoimmune diseases, congenital and idiopathic, in which neoplasm is the most common cause, whereas idiopathic cause comprises $6 \%-14 \%$ of all reported cases [2] [3]. Generally, the prognosis and severity of chylothorax depends upon the daily chyle output volume; either low $(<1000 \mathrm{~mL})$ or high $(\geq 1000 \mathrm{~mL})[4]$.

\section{Case Presentation}

A 38-year-old gentleman of Chinese ethnicity with no known medical illness, presented to the emergency department complaining of worsening dyspnoea of one-week duration, associated with mild pleuritic chest pain and non-productive cough. He denied other symptoms, including palpitation, dizziness, syncope or any fluid overload symptoms. Neither did he sustain any form of chest trauma nor did he complain of any constitutional symptoms. He also denied any recent travel history or sick contact. He does not have any family history of malignancy, pulmonary diseases or congenital heart disorder. He is an ex-smoker with 15-pack-years of smoking. He is also a social drinker but he denied usage of any prescribed medication or illicit drugs.

On admission, he was tachypneic with oxygen saturation of $96 \%$ under room air. Otherwise, he was afebrile and other vital signs were normal. Respiratory examination revealed reduced chest wall expansion, decreased vocal fremitus, stony dullness on percussion and reduced air entry on the right lung. Cardiovascular examination was unremarkable. The findings suggest a massive right sided pleural effusion, which was confirmed by an initial chest radiograph (Figure 1). An intercostal chest drain was inserted for symptomatic relief, draining a total of $1800 \mathrm{~mL}$ of pleural fluid over one week (Table 1). He was discharged after a ten-day admission. Over the span of three months, he was admitted multiple times due to similar complaints. As advanced and sophisticated tests are not readily available, he was referred to a tertiary centre before a lymphangiogram could be carried out.

Apart from a raised C-reactive protein (CRP) of $72.7 \mathrm{mg} / \mathrm{L}$, other routine blood investigations (FBC, LFT, UEC) were normal on presentation. Infective screenings, including Tuberculosis workup and viral screenings such as Human Immunodeficiency Virus (HIV), Hepatitis B and Hepatitis C antibodies were all negative. The pleural fluid from the chest tube was milky in appearance (Figure 2). Biochemical analysis of pleural fluid revealed total protein of $43.6 \mathrm{~g} / \mathrm{L}$ (Exudative: $>34.5 \mathrm{~g} / \mathrm{L})$, while serum protein was $69.0 \mathrm{~g} / \mathrm{L}(60-80 \mathrm{~g} / \mathrm{L})$, which is suggestive of exudative effusion based on Light's criteria. Pleural fluid lipid profile showed normal total cholesterol level of $2.63 \mathrm{mmol} / \mathrm{L}(<5.18 \mathrm{mmol} / \mathrm{L})$ and raised 
Table 1. Pleural fluid characteristics $(\mathrm{N}=$ Normal range) [5].

\begin{tabular}{cc}
\hline Appearance & Milky \\
\hline Amount & $1800 \mathrm{~mL}$ \\
Triglyceride & $12.67 \mathrm{mmol} / \mathrm{L}(\mathrm{N}:<1.24 \mathrm{mmol} / \mathrm{L})$ \\
Cholesterol & $2.63 \mathrm{mmol} / \mathrm{L}(\mathrm{N}:<5.18 \mathrm{mmol} / \mathrm{L})$ \\
Protein & $43.6 \mathrm{~g} / \mathrm{L}($ Exudative: $>34.5 \mathrm{~g} / \mathrm{L})$ \\
Cytology & Atypical small to medium lymphoid cells \\
Tuberculosis culture & No mycobacteria isolated \\
\hline
\end{tabular}

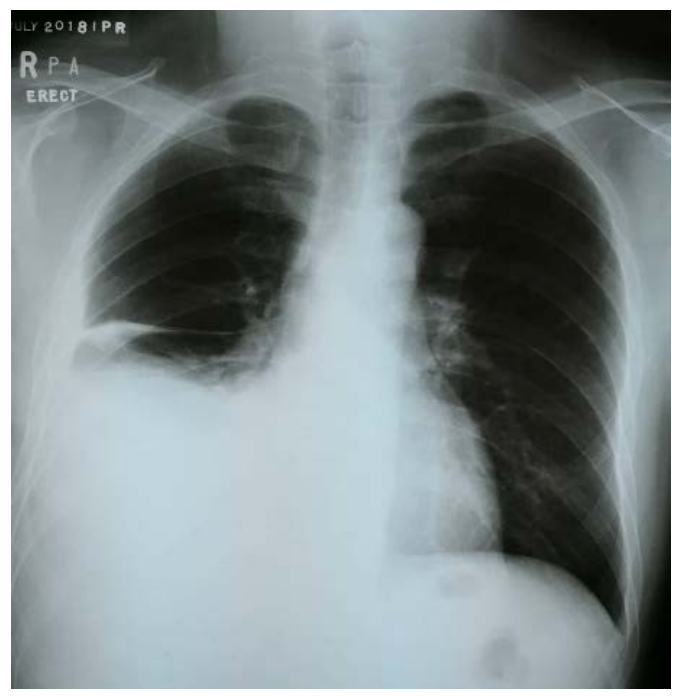

Figure 1. Right-sided pleural effusion to mid-zone.

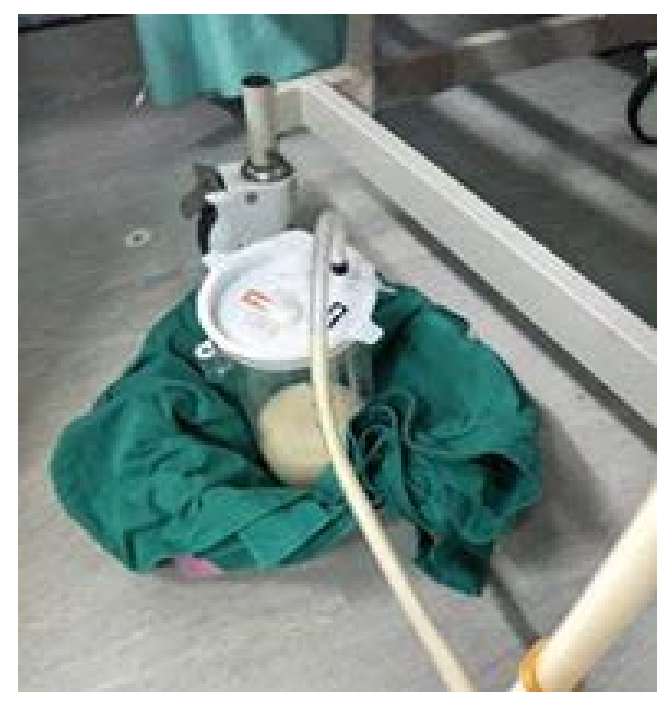

Figure 2. Milky appearance of pleural fluid.

triglyceride level of $12.67 \mathrm{mmol} / \mathrm{L}(<1.24 \mathrm{mmol} / \mathrm{L})$. Of note, serum triglyceride was only $0.86 \mathrm{mmol} / \mathrm{L}$.

Pleural fluid cytology analysis showed atypical small to medium lymphoid 
cells. In addition, pleural biopsy done was inconclusive as it revealed skeletal muscle bundle with fatty tissues and blood clot. Post thoracentesis, a contrast-enhanced computed tomography (CECT) scan showed no obvious mass or lymphadenopathy, which does not suggest malignancy, while the lymphangiogram, which was done in Hospital Kuala Lumpur, confirmed an extravasation of dye from the thoracic duct, likely at the T12 vertebra level. He was referred for thoracic duct ligation for his chylothorax in a tertiary centre.

\section{Discussion}

\subsection{Presentation}

Considering that chyle is a sterile fluid, patients would be relatively asymptomatic unless there is a large accumulation of chyle in the pleural cavity. Owing to a decimated inflammatory response mounted in the presence of chyle, fever and chest pain are uncommon clinical presentations [6]. Nevertheless, chyloptysis, which is an expectoration of chyle due to a formation of broncho-pleural fistula, has been reported, albeit a rare presenting complaint [7]. Bedside examination findings would be consistent with a typical pleural effusion which is usually unilateral (78\%) with a predilection of right hemi-thorax involvement (67\%) [8].

\subsection{Diagnosis}

In patients suspected of chylothorax, the primary diagnostic test is pleural fluid analysis. Pleural fluid samples are sent to assess for biochemistry, cytology, microbiology and lipid profile [5]. In most cases, the pleural fluid is exudative due to higher protein concentration [9] [10]. The diagnosis can be made confidently if the pleural fluid triglyceride exceeds $1.24 \mathrm{mmol} / \mathrm{L}$ and the cholesterol level is less than $5.18 \mathrm{mmol} / \mathrm{L}$. Triglyceride level below $0.56 \mathrm{mmol} / \mathrm{L}$ strongly excludes the diagnosis. Should the level falls between $0.56 \mathrm{mmol} / \mathrm{L}$ to $1.24 \mathrm{mmol} / \mathrm{L}$, pleural fluid lipoprotein electrophoresis is warranted to detect the presence of chylomicrons [9] [11] [12]. In this patient, chylothorax is diagnosed based on the milky appearance and exudative nature of the pleural fluid, along with high triglyceride and low cholesterol levels.

In order to identify the underlying cause, investigations which include peripheral eosinophil count for suspected filariasis and computed tomography (CT) should be performed [5]. In cases where the underlying cause remains uncertain, lymphatic imaging such as lymphangiography, lymphoscintigraphy and magnetic resonance (MR) lymphangiography may be performed to identify the location of lymph leakage [5].

\subsection{Conservative Management}

Essentially, conservative management entails three main principles, which are relieving symptoms, replenishing fluids and nutrients, and reducing chyle output [13]. Chest tube insertion will allow lung re-expansion and symptoms alle- 
viation. Since high-output chylothorax may result in an inordinate loss of nutrients, chestdrain output should be closely monitored and the replacement of the losses is imperative [13]. The reduction of chyle output could be achieved by introducing a high-protein and low-fat $(<10 \mathrm{~g} /$ day) diet [4]. Low-fat intake reduces intestinal fat absorption, therefore decreasing chyle flow which would eventually prevent chyle accumulation [4]. Besides, a medium-chain triglycerides (MCT) diet is effective in reducing the flow of chyle as these triglycerides are transported directly to the liver via portal venous system rather than being absorbed by the intestinal lymphatics [13] [14].

Apart from a diagnostic procedure, a lymphangiogram may occasionally be therapeutic due to the local-tissue sclerosing effect of the iodinated contrast. According to a case series study, lymphangiogram alleviated the symptoms of $70 \%$ and $35 \%$ of patients with low-output and high-output chylothorax respectively [4]. Adjunctive therapies such as somatostatin and a somatostatin analogue, subcutaneous octreotide can be used in patient with nonsurgical cause of chylothorax. Octreotide has been shown to inhibit secretions from gastric, pancreatic and biliary as well as inhibit absorption of chyle from the intestine, which would eventually decrease the volume of fluid in the thoracic duct [15] [16]. For patients who do not respond to conservative therapies, pleurodesis can be considered as an alternative, though limited studies have advocated the efficacy of pleurodesis alone without other treatment [4].

\subsection{Surgery vs Radiological Intervention}

Since the success rate of conservative management varies according to aetiologies and output volume, patients who showed poor response should be advised to proceed with definitive interventions, for instance thoracic duct ligation or embolization.

With regards to thoracic duct ligation, a lymphangiogram ought to be carried out to localise the area of thoracic leak and subsequently, depending on expertise available, the ligation can be performed through an open thoracotomy or a video-assisted thoracoscopy. In recent years, radiological intervention has been gaining popularity as it is less invasive in comparison to surgery. Limited studies have made direct comparison between surgery and radiological intervention. Nevertheless, it has been reported that thoracic duct ligation (up to 90\%) has a better success rate compared to an embolization (highest at 75\%), under the stipulation that both procedures were implemented successfully [8] [17] [18]. Despite the higher efficacy, a remarkable disparity was noted in the morbidity and mortality rates between surgery $(40 \%)$ and radiological intervention $(0 \%$ 2\%) [19]. However, considering that most studies also included many patients with post-operative chylothorax, the statistics should only serve as a guidance.

\section{Conclusion}

Managing chylothorax can be challenging in a rural setting due to the lack of 
relevant expertise and facilities. A timely diagnosis and treatment of chylothorax would require appropriate investigations and awareness of this rare medical condition. For adult patients suspected to have chylothorax, an underlying aetiology should be investigated as most cases are attributable to an underlying cause. Patients who are classified as low output chylothorax should commence on conservative management while awaiting for definitive treatment at a tertiary centre. High output chylothorax on the other hand, warrants an immediate referral to a tertiary centre.

\section{Consent}

Informed consent was obtained from the patient for the publication of this case report and the accompanying images.

\section{Acknowledgements}

None.

\section{Conflicts of Interest}

None.

\section{References}

[1] Chalret du Rieu, M., Baulieux, J., Rode, A. and Mabrut, J.Y. (2011) Management of Postoperative Chylothorax. Journal of Visceral Surgery, 148, e346-e352.

[2] Doerr, C.H., Allen, M.S., Nicholsv III, F.C. and Ryu J.H. (2005) Etiology of Chylothorax in 203 Patients. Mayo Clinic Proceedings, 80, 867-870.

https://doi.org/10.4065/80.7.867

[3] Hooper, C., Lee, Y.C., Maskell, N. and BTS Pleural Guideline Group (2010) Investigation of a Unilateral Pleural Effusion in Adults: British Thoracic Society Pleural Disease Guideline 2010. Thorax, 65, ii4. https://doi.org/10.1136/thx.2010.136978

[4] Heffner, J.E. (2018) Management of Chylothorax. Topic 16356 Version 17.0. https://www-uptodate-com.ezproxy.lib.monash.edu.au/contents/management-of-ch ylotho$\underline{\text { rax? }}$ search $=$ chylothorax $\&$ source $=$ search_result $\&$ selectedTitle $=2 \sim 68 \&$ usage_type $=\mathrm{d}$ efault\&display_rank=2

[5] Heffner, J.E. (2018) Etiology, Clinical Presentation, and Diagnosis of Chylothorax. Topic 6696 Version 17.0.

https://www-uptodate-com.ezproxy.lib.monash.edu.au/contents/etiology-clinical-pr esentation-and-diagnosis-of-chylothorax?search=chylothorax\&source=search_result\&sele ctedTitle $=1 \sim 68$ \&usage_type $=$ default\&display_rank $=1 \# \mathrm{H} 6$

[6] Prakash, U.B.S. (2002) Chylothorax and Pseudochylothorax. European Respiratory Monograph, 7, 249.

[7] Lim, K.G., Rosenow III, E.C., Staats, B., et al. (2004) Chyloptysis in Adults: Presentation, Recognition, and Differential Diagnosis. Chest, 125, 336-340. https://doi.org/10.1378/chest.125.1.336

[8] Maldonado, F., Cartin-Ceba, R., Hawkins, F.J. and Ryu, J.H. (2010) Medical and Surgical Management of Chylothorax and Associated Outcomes. The American 
Journal of the Medical Sciences, 339, 314-318.

https://doi.org/10.1097/MAJ.0b013e3181cdcd6c

[9] Maldonado, F., Hawkins, F.J., Daniels, C.E., et al. (2009) Pleural Fluid Characteristics of Chylothorax. Mayo Clinic Proceedings, 84, 129-133.

https://doi.org/10.4065/84.2.129

[10] Agrawal, V., Doelken, P. and Sahn, S.A. (2008) Pleural Fluid Analysis in Chylous Pleural Effusion. Chest, 133, 1436-1441. https://doi.org/10.1378/chest.07-2232

[11] Staats, B.A., Ellefson, R.D., Budahn, L.L., et al. (1980) The Lipoprotein Profile of Chylous and Nonchylous Pleural Effusions. Mayo Clinic Proceedings, 55, 700-704.

[12] Park, J.G., Aubry, M.C., Godfrey, J.A. and Midthun, D.E. (2006) Mediastinal Lymphangioma: Mayo Clinic Experience of 25 Cases. Mayo Clinic Proceedings, 81, 1197-1203. https://doi.org/10.4065/81.9.1197

[13] Lyon, S., Mott, N., Koukounaras, J., Shoobridge, J. and Hudson, P.V. (2013) Role of Interventional Radiology in the Management of Chylothorax: A Review of the Current Management of High Output Chylothorax. Cardiovascular and Interventional Radiology, 36, 599-607. https://doi.org/10.1007/s00270-013-0605-3

[14] Alejandre-Lafont, E., Krompiec, C., Rau, W.S. and Krombach, G.A. (2011) Effectiveness of Therapeutic Lymphography on Lymphatic Leakage. Acta Radiologica, 52, 305-311. https://doi.org/10.1258/ar.2010.090356

[15] Kalomenidis, I. (2006) Octreotide and Chylothorax. Current Opinion in Pulmonary Medicine, 12, 264-267. https://doi.org/10.1097/01.mcp.0000230629.73139.26

[16] Roehr, C.C., Jung, A., Proquitté, H., et al. (2006) Somatostatin or Octreotide as Treatment Options for Chylothorax in Young Children: A Systematic Review. Intensive Care Medicine, 32, 650-657. https://doi.org/10.1007/s00134-006-0114-9

[17] Paul, S., Altorki, N.K., Port, J.L., et al. (2009) Surgical Management of Chylothorax. The Thoracic and Cardiovascular Surgeon, 57, 226-228. https://doi.org/10.1055/s-0029-1185457

[18] Nadolski, G.J. and Itkin, M. (2013) Thoracic Duct Embolization for Nontraumatic Chylous Effusion: Experience in 34 Patients. Chest, 143, 158-163. https://doi.org/10.1378/chest.12-0526

[19] Cerfolio, R.J., Allen, M.S., Deschamps, C., et al. (1996) Postoperative Chylothorax. The Journal of Thoracic and Cardiovascular Surgery, 112, 1361-1366. https://doi.org/10.1016/S0022-5223(96)70152-6 